\title{
A Note on Two-Agent Scheduling with Resource Dependent Release Times on a Single Machine
}

\author{
Peng Liu ${ }^{1}$ and Lini Duan ${ }^{2}$ \\ ${ }^{1}$ School of Management, Shenyang University of Technology, Shenyang 110870, China \\ ${ }^{2}$ School of Economics \& Management, Shenyang University of Chemical Technology, Shenyang 110142, China \\ Correspondence should be addressed to Peng Liu; liup7802@163.com
}

Received 6 October 2014; Accepted 30 November 2014

Academic Editor: Shuenn-Ren Cheng

Copyright (c) 2015 P. Liu and L. Duan. This is an open access article distributed under the Creative Commons Attribution License, which permits unrestricted use, distribution, and reproduction in any medium, provided the original work is properly cited.

\begin{abstract}
We consider a scheduling problem in which both resource dependent release times and two agents exist simultaneously. Two agents share a common single machine, and each agent wants to minimize a cost function dependent on its own jobs. The release time of each $A$-agent's job is related to the amount of resource consumed. The objective is to find a schedule for the problem of minimizing $A$-agent's total amount of resource consumption with a constraint on $B$-agent's makespan. The optimal properties and the optimal polynomial time algorithm are proposed to solve the scheduling problem.
\end{abstract}

\section{Introduction}

Machine scheduling problems with multiagent have received increasing attention in recent years. Different agents share a common processing machine, and each agent wants to minimize a cost function depending on its jobs only. Scheduling with multiple agents is firstly introduced by Baker and Smith [1] and Agnetis et al. [2]. Baker and Smith [1] consider the problem of minimizing a convex combination of the agents' objective functions. They provide some dominance properties and demonstrate where the problem becomes computationally difficult. Agnetis et al. [2] address the scheduling problem in which two agents compete for the usage of shared processing resource and each agent has his own criterion to optimize. They study the constrained optimization problem and the Pareto-optimization problem on a single machine and the shop environment. Agnetis et al. [3] analyze the complexity of some multiagent scheduling problems on a single machine and propose solution algorithms. Lee et al. [4] provide approximation algorithms for multiagent scheduling to minimize total weighted completion time. Gawiejnowicz et al. [5] consider a single-machine two-agent scheduling problem with proportionally deteriorating job processing times. Yin et al. [6] consider several two-agent scheduling problems with assignable due dates on a single machine. Cheng et al. [7] study a two-agent single-machine scheduling problem with release times to minimize the total weighted completion time. Yu et al. [8] investigate several single-machine two-synergetic-agent scheduling problems. Yin et al. [9] consider two-agent single-machine scheduling problems with unrestricted due date assignment.

The scheduling problems with resource consumption have been studied for many years. Cheng and Janiak [10] study the resource-optimal control of job completion on a single machine with a constraint on maximum job completion time. Vasilev and Foote [11] investigate a single machine scheduling problem where the resource consumed depends on the release times of jobs. Kaspi and Shabtay [12] consider the problem of scheduling jobs on a single machine where job-processing times are controllable through the allocation of a common limited resource. Wang and Cheng [13] consider the single machine scheduling problem with resource dependent release times and processing times. Wei et al. [14] consider the single-machine scheduling with time-and-resourcedependent processing times. J.-B. Wang and M.-Z. Wang [15] consider the single-machine scheduling to minimize total convex resource consumption with a constraint on total weighted flow time. Yin et al. [16] consider single-machine due window assignment and scheduling problems with a common flow allowance and controllable job processing 
time. Lu et al. [17] consider a single-machine earlinesstardiness scheduling problem with due-date assignment, in which the processing time of a job is a function of its position in a sequence and its resource allocation.

However, to the best of our knowledge, no work has been done on models with both aspects of resource dependent release times and multiagent in the literature. These two categories of scheduling problems have been extensively and separately researched over the last two decades. In this paper, we study the two-agent scheduling problems on a single machine with resource dependent release times, where the goal is to find a schedule that minimizes the objective function of one agent with the restriction that the objective function of the other agent cannot exceed a given bound. The problems under consideration fall into the category of scheduling problems with resource consumption and multiple agents. Such a scheduling problem commonly arises in the steel industry. Janiak [18] describes a practical scheduling problem with resource dependent release times in steel mills, where batches of ingots have to be preheated before they can be hot rolled in a blooming mill, and the ingot preheating time is inversely proportional to the total amount of resources consumed.

The remainder of this paper is organized as follows. In Section 2, we describe the proposed problem. In Section 3, we develop the optimal polynomial time algorithm for the twoagent single-machine scheduling problem. Section 4 gives some concluding remarks.

\section{Problem Description}

We now describe our problem formally. There are two families of independent and nonpreemptive jobs $J^{A}=\left\{J_{1}^{A}, J_{2}^{A}, \ldots\right.$, $\left.J_{n_{A}}^{A}\right\}$ and $J^{B}=\left\{J_{1}^{B}, J_{2}^{B}, \ldots, J_{n_{B}}^{B}\right\}$ to be processed on a common single machine. The jobs in $J^{A}$ and $J^{B}$ are called $A$-agent's jobs and $B$-agent's jobs, respectively. Associated with each job $J_{h}^{A}$, let $p_{h}^{A}$ denote the processing time and $h=1,2, \ldots, n_{A}$. The release time $r_{h}^{A}$ is related to the amount of the resource $f_{h}^{A}$, consumed on job $J_{h}^{A}$. A strictly decreasing continuous function is given $f: R^{+} \rightarrow R^{+}$. We refer to $f$ as the resource consumption function. We assume that $f_{h}^{A}=f\left(r_{h}^{A}\right)$. Each $A$-agent's job can start at any time after the release time of the job, and idle time between jobs is allowed. Since the consumption function $f$ is strictly decreasing continuously, we may assume that each job starts as soon as it becomes available. That is, we can take $s_{h}^{A}=r_{h}^{A}$, where $s_{h}^{A}$ is the starting time of job $J_{h}^{A}$. Associated with each job $J_{k}^{B}$, let $p_{k}^{B}$ and $r_{k}^{B}$ denote the processing time and the release times, respectively, and $k=1,2, \ldots, n_{B}$. Let $\pi$ indicate a feasible schedule of the $n=n_{A}+n_{B}$ jobs. Let $C_{k}^{B}(\pi)$ denote the completion time of $B$-agent's job $J_{k}^{B}$ under schedule $\pi$. The objective function of agent $A$ is to minimize the total amount of resource consumption $\sum_{h=1}^{n_{A}} f\left(r_{h}^{A}\right)$. The objective function of agent $B$ is to minimize the makespan $C_{\max }^{B}=\max _{k=1,2, \ldots, n_{B}}\left\{C_{k}^{B}(\pi)\right\}$.

The goal is to minimize the total amount of resource consumption $\sum_{h=1}^{n_{A}} f\left(r_{h}^{A}\right)$ of agent $A$ with the restriction that the makespan $C_{\max }^{B}$ of agent $B$ cannot exceed a given bound $U$. If the value $U$ is too small, an instance of the scheduling problem may not have feasible solutions. If there is at least one feasible solution, we say that the instance is feasible. According to the three-field notation $\psi_{1}\left|\psi_{2}\right| \psi_{3}$ of Graham et al. [19], the scheduling problem is denoted as $1 \| \sum_{h=1}^{n_{A}} f\left(r_{h}^{A}\right)$ : $C_{\max }^{B} \leq U$.

\section{Main Results}

In this section, we develop an optimal polynomial time algorithm to solve the problem $1 \| \sum_{h=1}^{n_{A}} f\left(r_{h}^{A}\right): C_{\max }^{B} \leq U$.

Given a sequence $\pi=\left\{J_{1}^{B}, J_{2}^{B}, \ldots, J_{n_{B}}^{B}, J_{1}^{A}, J_{2}^{A}, \ldots, J_{n_{A}}^{A}\right\}$, for each $B$-agent' job, the completion time $C_{k}^{B}$ may be completed recursively as $C_{1}^{B}=r_{1}^{B}+p_{1}^{B}, C_{k}^{B}=\max \left\{r_{k}^{B}, C_{k-1}^{B}\right\}+p_{k}^{B}, k=$ $2, \ldots, n_{B}$.

Thus the completion time of job $J_{k}^{B}$ may also be taken as $C_{k}^{B}=\max _{1 \leq j \leq k}\left\{r_{j}^{B}+\sum_{l=j}^{k} p_{l}^{B}\right\}$.

Moreover, let the maximum job completion time be denoted by $C_{\max }^{B}=\max _{1 \leq k \leq n_{B}}\left\{C_{k}^{B}\right\}$; then $C_{\max }^{B}=$ $\max _{1 \leq k \leq n_{B}}\left\{r_{k}^{B}+\sum_{i=k}^{n_{B}} p_{i}^{B}\right\}$.

Lemma 1. Given a sequence $\pi=\left\{J_{1}^{B}, J_{2}^{B}, \ldots, J_{n_{B}}^{B}, J_{1}^{A}, J_{2}^{A}, \ldots\right.$, $\left.J_{n_{A}}^{A}\right\}$ and a constant $U$, define $C_{\pi}^{B}=\max _{1 \leq k \leq n_{B}}\left\{r_{k}^{B}+\sum_{i=k}^{n_{B}} p_{i}^{B}\right\}$. Then, if $U<C_{\pi}^{B}$, the sequence $\pi$ corresponds to an infeasible schedule.

Now we can define bounds for the constraint $U$. Define $\underline{U}=\max _{1 \leq k \leq n_{B}}\left\{r_{k}^{B}+\sum_{i=k}^{n_{B}} p_{i}^{B}\right\}$. The analysis in the following section will be confined to the case in which $\underline{U} \leq U$.

Lemma 2. An optimal schedule exists in which the A-agent's jobs are processed in the nonincreasing order of processing times $p_{h}^{A}$.

Proof. The resource consumption function $f$ is a strictly decreasing continuous function to $A$-agent's jobs. Since releasing $A$-agent's jobs sooner consumes more resource, $A$ agent's jobs should be released as late as possible. Hence $A$ agent's jobs should be released in nonincreasing order of $p_{h}^{A}$.

Lemma 3. An optimal schedule exists in which the B-agent's jobs are processed in the nondecreasing order of release times $r_{k}^{B}$

Proof. The makespan of agent $B$ is the maximum completion time of $B$-agent's jobs on the single machine; that is, the makespan of agent $B$ is the completion time of the last $B$ agent's job. Using a pairwise job interchange argument, we can process $B$-agent's jobs in the nondecreasing order of release times $r_{k}^{B}$.

Next, an algorithm to determine an optimal schedule of the problem $1 \| \sum_{h=1}^{n_{A}} f\left(r_{h}^{A}\right): C_{\max }^{B} \leq U$ is developed as follows. 
Algorithm 4.

Step 1. Arrange the $A$-agent's jobs as $\left\{J_{1}^{A}, J_{2}^{A}, \ldots, J_{n_{A}}^{A}\right\}$ according to the nonincreasing order of $p_{h}^{A}$ and denote all $B$-agent's jobs sequenced by the nondecreasing order of $r_{k}^{B}$ as a dummy job $B 1$.

Step 2. Define sequence $S=\left\{B 1, J_{1}^{A}, J_{2}^{A}, \ldots, J_{n_{A}-1}^{A}, J_{n_{A}}^{A}\right\}$ and calculate $C_{\max }^{B}$ for agent $B$. The sequence $S$ is an optimal schedule and the starting times of $A$-agent's job are given by $s_{1}^{A}=U, s_{h}^{A}=s_{h-1}^{A}+p_{h-1}^{A}=s_{1}^{A}+\sum_{i=1}^{h-1} p_{i}^{A}, h=2,3, \ldots, n_{A}$.

Theorem 5. Algorithm 4 generates an optimal schedule for the problem $1 \| \sum_{h=1}^{n_{A}} f\left(r_{h}^{A}\right): C_{\max }^{B} \leq U$ in $O\left(n_{A} \log n_{A}+n_{B} \log n_{B}\right)$ time.

Proof. The proof of optimality is straightforward from the results of Lemmas 1-3. We now turn to time complexity. The time to sequence the jobs of set $J^{A}$ according to the nonincreasing order of $p_{h}^{A}$ is $O\left(n_{A} \log n_{A}\right)$. The time to sequence the jobs of set $J^{B}$ according to the nondecreasing order of $r_{k}^{B}$ is $O\left(n_{B} \log n_{B}\right)$. Creating dummy job $B 1$ incurs $O\left(n_{B}\right)$ operations. So the overall computational complexity of Algorithm 4 is bounded by $O\left(n_{A} \log n_{A}+n_{B} \log n_{B}\right)$. This completes the proof.

\section{Conclusions}

In this paper, we combine two important issues in scheduling that recently have received increasing attention from researchers: resource dependent release times and multiple agents. Our goal is to find a schedule for the problem of minimizing $A$-agent's total amount of resource consumption with a constraint on $B$-agent's makespan. We propose the optimal properties and the optimal polynomial time algorithm for the considered scheduling problem.

The future research may be directed to analyze the problems with other objective functions such as minimizing the number of late jobs, the total weighted completion time and tardiness. An interesting research topic is also to analyze the scheduling problem with more than two agents or in other machine environments.

\section{Conflict of Interests}

The authors declare that there is no conflict of interests regarding the publication of this paper.

\section{Acknowledgments}

The authors are grateful to the editor and reviewers for their helpful comments and suggestions. This research is supported by the National Natural Science Foundation of China (Grant no. 71001074) and the Program for Liaoning Excellent Talents in University (Grant no. WJQ2013003).

\section{References}

[1] K. R. Baker and J. C. Smith, "A multiple-criterion model for machine scheduling," Journal of Scheduling, vol. 6, no. 1, pp. 716, 2003.

[2] A. Agnetis, P. B. Mirchandani, D. Pacciarelli, and A. Pacifici, "Scheduling problems with two competing agents," Operations Research, vol. 52, no. 2, pp. 229-242, 2004.

[3] A. Agnetis, D. Pacciarelli, and A. Pacifici, "Multi-agent single machine scheduling," Annals of Operations Research, vol. 150, no. 1, pp. 3-15, 2007.

[4] K. Lee, B.-C. Choi, J. Y. Leung, and M. L. Pinedo, "Approximation algorithms for multi-agent scheduling to minimize total weighted completion time," Information Processing Letters, vol. 109, no. 16, pp. 913-917, 2009.

[5] S. Gawiejnowicz, W.-C. Lee, C.-L. Lin, and C.-C. Wu, "Singlemachine scheduling of proportionally deteriorating jobs by two agents," Journal of the Operational Research Society, vol. 62, no. 11, pp. 1983-1991, 2011.

[6] Y. Yin, S.-R. Cheng, T. C. Cheng, C.-C. Wu, and W.-H. Wu, "Two-agent single-machine scheduling with assignable due dates," Applied Mathematics and Computation, vol. 219, no. 4, pp. 1674-1685, 2012.

[7] T. C. Cheng, Y. H. Chung, S. C. Liao, and W. C. Lee, "Twoagent singe-machine scheduling with release times to minimize the total weighted completion time," Computers and Operations Research, vol. 40, no. 1, pp. 353-361, 2013.

[8] X. Yu, Y. Zhang, D. Xu, and Y. Yin, "Single machine scheduling problem with two synergetic agents and piece-rate maintenance," Applied Mathematical Modelling: Simulation and Computation for Engineering and Environmental Systems, vol. 37, no. 3, pp. 1390-1399, 2013.

[9] Y. Q. Yin, T. C. E. Cheng, X. Q. Yang, and C. C. Wu, “Twoagent single-machine scheduling with unrestricted due date assignment," Computers \& Industrial Engineering, vol. 79, pp. 148-155, 2014.

[10] T. C. Cheng and A. Janiak, "Resource optimal control in some single-machine scheduling problems," IEEE Transactions on Automatic Control, vol. 39, no. 6, pp. 1243-1246, 1994.

[11] S. H. Vasilev and B. L. Foote, "On minimizing resource consumption with constraints on the makespan and the total completion time," European Journal of Operational Research, vol. 96, no. 3, pp. 612-621, 1997.

[12] M. Kaspi and D. Shabtay, "Convex resource allocation for minimizing the makespan in a single machine with job release dates," Computers and Operations Research, vol. 31, no. 9, pp. 1481-1489, 2004.

[13] X. Wang and T. C. Cheng, "Single machine scheduling with resource dependent release times and processing times," European Journal of Operational Research, vol. 162, no. 3, pp. 727-739, 2005.

[14] C.-M. Wei, J.-B. Wang, and P. Ji, "Single-machine scheduling with time-and-resource-dependent processing times," Applied Mathematical Modelling: Simulation and Computation for Engineering and Environmental Systems, vol. 36, no. 2, pp. 792-798, 2012.

[15] J.-B. Wang and M.-Z. Wang, "Single-machine scheduling to minimize total convex resource consumption with a constraint on total weighted flow time," Computers \& Operations Research, vol. 39, no. 3, pp. 492-497, 2012. 
[16] Y. Yin, T. C. E. Cheng, C.-C. Wu, and S.-R. Cheng, "Singlemachine due window assignment and scheduling with a common flow allowance and controllable job processing time," Journal of the Operational Research Society, vol. 65, no. 1, pp. 113, 2014.

[17] Y.-Y. Lu, G. Li, Y.-B. Wu, and P. Ji, “Optimal due-date assignment problem with learning effect and resource-dependent processing times," Optimization Letters, vol. 8, no. 1, pp. 113-127, 2014.

[18] A. Janiak, "Minimization of the blooming mill standstillsmathematical model, suboptimal algorithms," Mechanika, vol. 8, no. 2, pp. 37-49, 1989.

[19] R. L. Graham, E. L. Lawler, J. K. Lenstra, and A. H. R. Kan, "Optimization and approximation in deterministic sequencing and scheduling theory: a survey," Annals of Discrete Mathematics, vol. 5, pp. 287-326, 1979. 


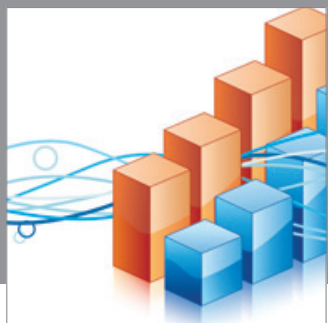

Advances in

Operations Research

mansans

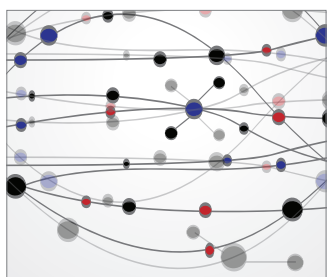

The Scientific World Journal
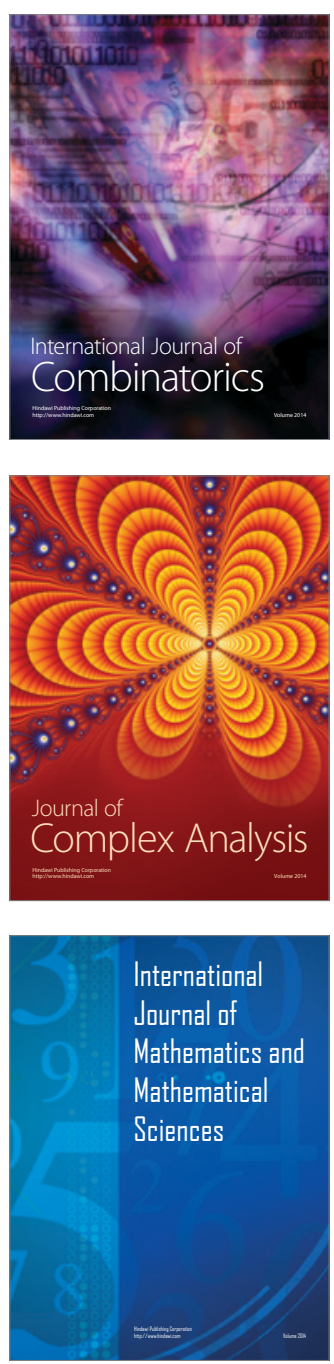
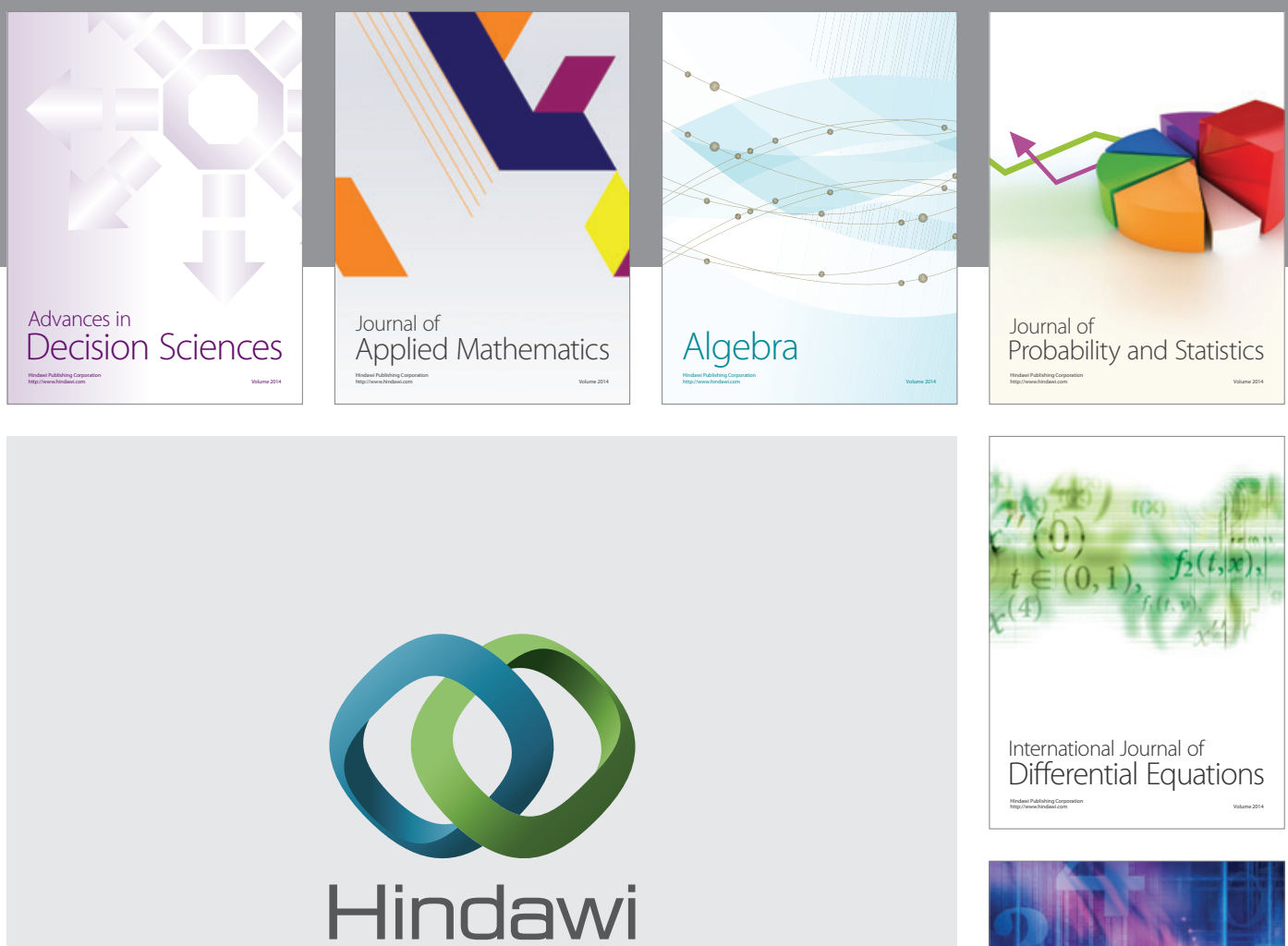

Submit your manuscripts at http://www.hindawi.com
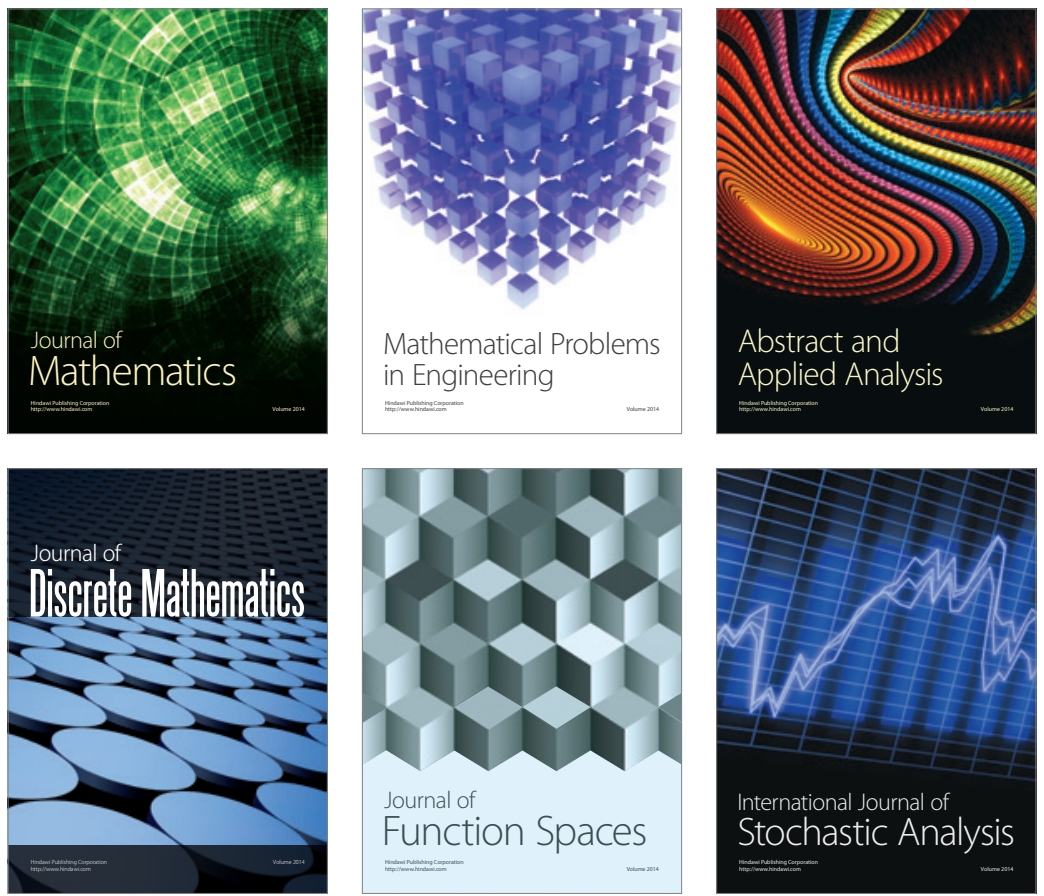

Journal of

Function Spaces

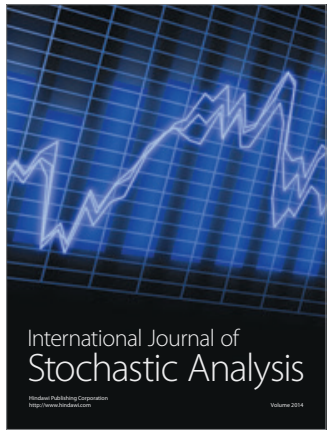

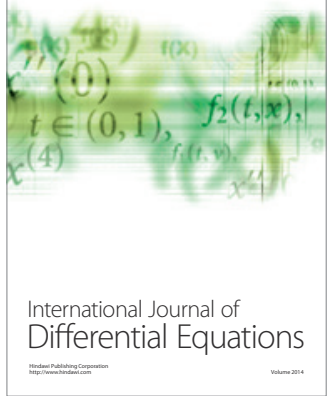
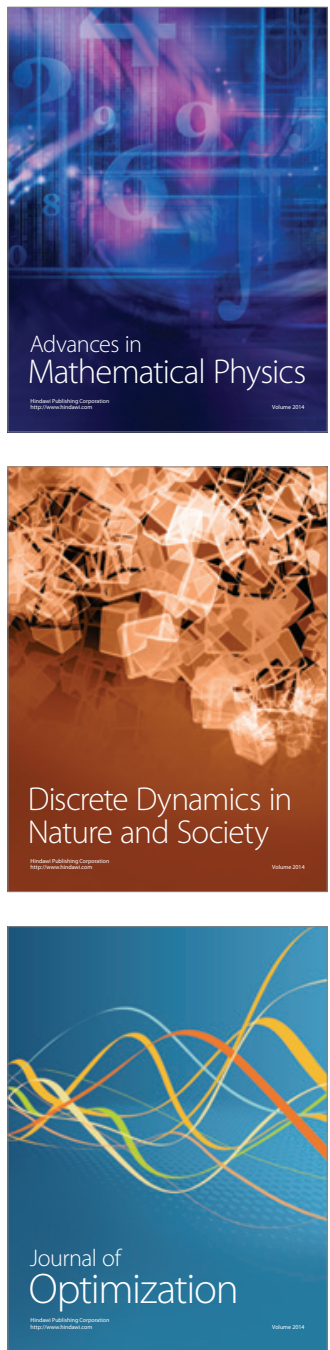